\title{
ON THE MECHANICAL BEHAVIOUR OF METALS IN THE STRAIN-HARDENING RANGE*
}

\author{
BY \\ G. H. HANDELMAN, C. C. LIN AND W. PRAGER \\ Brown University
}

1. Introduction. The present paper is concerned with certain stress-strain relations purporting to describe the mechanical behaviour of quasi-isotropic metals in the strain-hardening range. As a preparation for a more precise characterization of these relations, let us consider the tension test of a metal like copper or aluminum which does not flow under a constant stress, but exhibits strain hardening. If the test involves loading only, i.e., if the reduced tensile stress ${ }^{1} \sigma$ or the tensile strain $\epsilon$ increase throughout the test, the resulting diagram of reduced stress versus strain will have the general appearance of the curve $O P Q$ in Fig. 1. On the other hand, if the test specimen is unloaded after a certain point, such as $P$, has been reached along this curve, the stress-strain diagram for unloading is found to be very nearly a straight line $P A$ which is parallel to the tangent of the curve $O P Q$ at $O$. After complete unloading, the specimen shows a permanent extension which corresponds to the permanent strain represented by $O A$.

To simplify the discussion, let us assume at present that the material is incompressible. A longitudinal extension $\epsilon$ of the isotropic specimen is then ac-

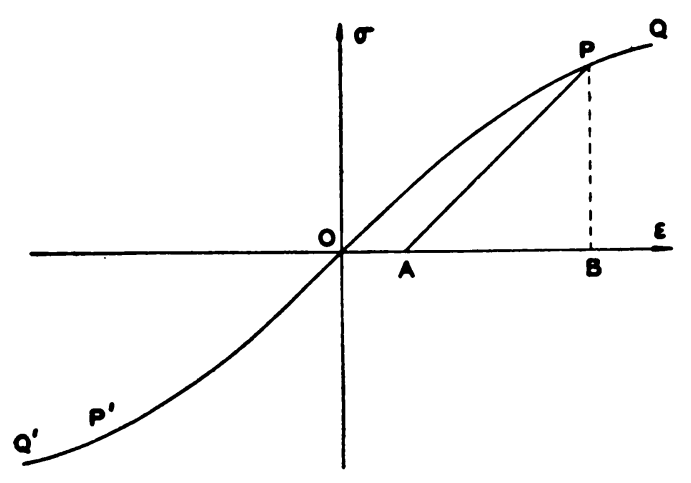

FIg. 1. Typical curve of reduced stress vs. strain. companied by a uniform lateral contraction of the magnitude $\epsilon / 2$. If the discussion is restricted to states of stress and strain which can be reached by a single loading followed by one complete or partial unloading at the most, the mechanical behaviour of the material in simple tension is therefore completely defined by the curve $O P Q$. It will be assumed in the following that for the materials under consideration the stress-strain diagram in simple compression $\left(O P^{\prime} Q^{\prime}\right.$ in Fig. 1) is obtained by reflecting the curve $O P Q$ with respect to the origin $O$, and that the practically important portion of the curve $Q^{\prime} O Q$, i.e., the portion corresponding to small and moderate strains, is represented with sufficient accuracy by a development of the form

$$
\epsilon=\sigma+a_{3} \sigma^{3}+a_{5} \sigma^{5}+\cdots,
$$

where $a_{3}, a_{5}, \ldots$ are constants. (The coefficient of the linear term on the right-hand side of (1) must be unity since $\sigma$ is the reduced stress. No even powers of $\sigma$ can occur

* Received September 17, 1946.

1 The reduced stress is defined as the quotient of the stress by Young's modulus. 
on the right-hand side of (1), because the stress-strain diagrams for tension and compression are assumed to be congruent.)

In the case of simple tension or compression, the mechanical behaviour of the material during the first loading is readily represented by a finite relation of the form (1); the behaviour during the first unloading, however, is most naturally represented by the differential stress-strain relation

$$
d \epsilon=d \sigma,
$$

for this form avoids explicit reference to the state of stress at which the unloading began. Accordingly, it is of ten convenient to write Eq. (1), too, in differential form:

$$
d \epsilon=\alpha(\sigma) d \sigma .
$$

Here, $\alpha(\sigma)=d \epsilon / d \sigma=1+3 a_{3} \sigma^{2}+5 a_{5} \sigma^{4}+\cdots$ equals the quotient of Young's modulus by the so-called tangent modulus. To arrive at a complete analytical description of the mechanical behaviour of the material in simple tension and compression, we must supplement the preceding equations by analytical criteria for loading and unloading. For tension $(\sigma>0)$ loading corresponds to $d \sigma>0$ and unloading to $d \sigma<0$; for compression $(\sigma<0)$ these criteria must be reversed. A satisfactory criterion for loading and unloading is therefore furnished by the sign of $\sigma d \sigma=d\left(\frac{1}{2} \sigma^{2}\right)$.

The present paper is concerned with the extension of this analysis to general states of stress and strain which can be reached by a single loading followed at most by one complete or partial unloading. In the case of simple tension or compression, a differential stress-strain relation of the form (3) which is valid for the first loading can always be integrated under the initial condition $\epsilon=0$ for $\sigma=0$ and is thus equivalent to a finite stress-strain relation. For more general states of stress, however, a suitably generalized form of the differential stress-strain relation (3) may be in tegrable or not. The distinction between differential and finite stress-strain relations for the first loading is therefore no longer a purely formal matter, but acquires physical significance. One of the main results of the following discussion consists in the remark that the assumption of a finite stress-strain relation for the first loading is incompatible with certain postulates concerning the mechanical behaviour under those changes of stress which constitute neither loading nor unloading. This is shown in Section 3. Sections 2 and 4 are devoted to the discussion of finite and differential stress-strain relations, respectively. Section 5 gives a method of correlating experimental results with the present theory. Finally, Section 6 contains a discussion of the limitations of the theory.

2. Finite stress-strain relations. Using rectangular Cartesian coordinates $x_{i}$, $(i=1,2,3)$, we denote the displacement from the standard state by $u_{i}$, the strain by $\epsilon_{i j}$ and the reduced stress by $\sigma_{i j}$. For the small deformations to which the following discussion is restricted, the strain $\epsilon_{i j}$ is given by

$$
\epsilon_{i j}=\frac{1}{2}\left(u_{i, j}+u_{j, i}\right)
$$

where $u_{i, j}$ stands for $\partial u_{i} / \partial x_{j}$, etc. Adopting the usual summation convention regarding repeated subscripts, we define the mean normal strain as

and the strain deviation as

$$
e=\frac{1}{3} \epsilon_{i i},
$$

$$
e_{i j}=\epsilon_{i j}-e \delta_{i j}
$$


where $\delta_{i j}$ is the Kronecker delta. Similarly, the reduced mean normal stress $s$ and the deviation $s_{i j}$ of the reduced stress are defined as

$$
s=\frac{1}{3} \sigma_{i i}
$$

and

$$
s_{i j}=\sigma_{i j}-s \delta_{i j} .
$$

According to the definitions of the deviations $e_{i j}$ and $s_{i j}$, we have

$$
e_{i i}=0, \quad s_{i i}=0 .
$$

The task of generalizing the finite stress-strain relation (1) is simplified by the remark that the first term on the right-hand side represents that part of the total strain $\epsilon$ which is recovered upon complete unloading. The remaining terms on the righthand side of (1) accordingly represent the permanent strain. In Fig. 1 the total strain is represented by the segment $O B$, the recoverable strain by $A B$, and the permanent strain by $O A$.

Setting

$$
\epsilon_{i j}=\epsilon_{i j}^{\prime}+\epsilon_{i j}^{\prime \prime},
$$

where $\epsilon_{i j}^{\prime}$ denotes the recoverable and $\epsilon_{i j}^{\prime \prime}$ the permanent strain, we may assume that the recoverable strain is related to the reduced stress by means of the generalized law of Hooke:

$$
\epsilon_{i j}^{\prime}=(1+\nu) s_{i j}+(1-2 \nu) s \delta_{i j} .
$$

Here $\nu$ denotes Poisson's ratio. We are then left with the task of supplementing (11) by a relation which expresses the permanent strain occurring during the first loading in terms of the reduced stress. For an isotropic material, this relation can only contain scalar constants in addition to the tensors $\epsilon_{i j}^{\prime \prime}, \sigma_{i j}$ and $\delta_{i j}$, and their invariants. Furthermore, the principal axes of $\epsilon_{i j}^{\prime \prime}$ and $\sigma_{i j}$ must coincide. Under the pressures commonly encountered in the testing of materials, no permanent change of volume is observed, i.e., $\epsilon_{i \mathfrak{i}}^{\prime \prime}=0$ and $\epsilon_{i j}^{\prime \prime}=e_{i j}^{\prime \prime}$. A state of hydrostatic pressure therefore does not produce any permanent strain, and two states of stress which differ only by a state of hydrostatic pressure may be expected to produce identical permanent strains. The permanent strain $\epsilon_{i j}^{\prime \prime}$ is thus independent of $s$ and depends only on the deviation $s_{i j}$. Furthermore, if the stress-strain diagrams for simple tension and simple compression are congruent, a reversal of the signs of all stresses may be expected to produce a mere reversal of the signs of all principal strains. Finally, if the ratios of the principal stresses are kept constant during the loading process, the ratios of the principal permanent strains, too, can be expected to remain constant.

In a recent paper, ${ }^{2} \mathrm{~W}$. Prager established the most general stress-strain relation which is compatible with the preceding postulates. With the notations

$$
J_{2}=\frac{1}{2} s_{i j} s_{j i}, \quad J_{3}=\frac{1}{3} s_{i j} s_{j k} s_{k i},
$$

and

$$
t_{i j}=s_{i k} s_{k j}-\frac{2}{3} J_{2} \delta_{i j}
$$

Prager's stress-strain relation can be written in the form

2 W. Prager, Strain-hardening under combined stresses, J. Appl. Phys. 16, 837-840 (1945). 


$$
\epsilon_{i j}^{\prime \prime}=F\left(J_{2}, J_{3}^{2}\right)\left[P\left(J_{2}, J_{3}^{2}\right) s_{i j}+Q\left(J_{2}, J_{3}^{2}\right) J_{3} t_{i j}\right],
$$

where $P$ and $Q$ must be homogeneous in the components of the stress deviation, the degree of $P$ exceeding that of $Q$ by 4 . The expressions (12) are second and third order invariants of the stress deviation $s_{i j}$ (the first order invariant $s_{i i}$ vanishes). The tensor (13) is the deviation of the square $s_{i k} s_{k j}$ of the stress deviation $s_{i j}$.

Combining (11) and (14), we obtain the desired generalization of the finite stressstrain relation $(1)$ :

$$
\epsilon_{i j}=(1+\nu) s_{i j}+(1-2 \nu) s \delta_{i j}+F\left(J_{2}, J_{3}^{2}\right)\left[P\left(J_{2}, J_{3}^{2}\right) s_{i j}+Q\left(J_{2}, J_{3}^{2}\right) J_{3} t_{i j}\right] .
$$

3. Neutral changes of stress. Inadmissibility of finite stress-strain relations. In the case of simple tension or compression the sign of $\sigma d \sigma=d\left(\frac{1}{2} \sigma^{2}\right)$ proved to be a satisfactory criterion for loading and unloading. Accordingly, one might consider the possibility of using the sign of $\sigma_{j i} d \sigma_{j i}$ as a criterion in the general case. If, however, the term "loading" is reserved for such changes of stress which are accompanied by a change of the permanent strain, this criterion is not satisfactory. Indeed, on account of (8) and the second $\mathrm{Eq}$. (9), we have

$$
\sigma_{i j} d \sigma_{i j}=\left(s_{i j}+s \delta_{i j}\right)\left(d s_{i j}+d s \delta_{i j}\right)=s_{i j} d s_{i j}+3 s d s .
$$

If loading were to correspond to $\sigma_{i j} d \sigma_{i j}>0$, a change of stress for which $d s_{i j}=0$ might therefore constitute loading in spite of the fact that such a change of stress is not accompanied by a change of the permanent strain. To avoid this difficulty, we shall use the sign of $s_{i j} d s_{i j}=d J_{2}$ as the desired criterion, an increase of $J_{2}$ corresponding to loading, a decrease to unloading.

Whereas for uniaxial stress any change of stress constitutes either loading or unloading, we have three kinds of change of stress in the general case, according to whether $J_{2}$ increases, remains constant, or decreases. An infinitesimal change of stress for which $d J_{2}=0$, will be called a neutral change of stress. For instance, any change of stress which affects only the mean normal stress, but leaves the stress deviation untouched, is a neutral change of stress. A more interesting example of a neutral change of stress is given by

$$
\sigma_{i j}=\left(\begin{array}{lll}
\sigma & 0 & 0 \\
0 & 0 & 0 \\
0 & 0 & 0
\end{array}\right), \quad d \sigma_{i j}=\left(\begin{array}{lll}
0 & d \tau & 0 \\
d \tau & 0 & 0 \\
0 & 0 & 0
\end{array}\right) .
$$

Equation (17) represents the stress system which arises from a combined tension and torsion test of a thin walled circular cylinder. Specifically, consider such a test piece which is pulled to an arbitrary tensile stress $\sigma$. If the traction is then kept constant and a small torque applied, the resulting systems of stress and increments of stress are represented by Eq. (17).

Let us now suppose that for the first loading $\left(d J_{2}>0\right)$ we have the finite stressstrain relation (15) and for unloading $\left(d J_{2}<0\right)$ the generalized-law of Hooke in the differential form

$$
d \epsilon_{i j}=(1+\nu) d s_{i j}+(1-2 \nu) d s \delta_{i j} .
$$

The simultaneous use of the stress-strain relations (15) and (18) will lead to obvious difficulties, unless these relations give identical strain increments for neutral changes 
of stress. We shall show that, in general, this continuity condition is not fulfilled. Indeed, if (15) is written in differential form, the first two terms on the right-hand side equal the right-hand side of (18); the continuity condition therefore requires the vanishing of the remaining terms on the right-hand side of the differential form of Eq. (15). Consider, for example, the stress and increment of stress given by Eq. (17). A simple computation will show that

$$
\begin{array}{rlrl}
s_{i j} & =\left(\begin{array}{lcc}
\frac{2}{3} \sigma & 0 & 0 \\
0 & -\frac{1}{3} \sigma & 0 \\
0 & 0 & -\frac{1}{3} \sigma
\end{array}\right), & t_{i j} & =\left(\begin{array}{lll}
\frac{2}{3} \sigma^{2} & 0 & 0 \\
0 & -\frac{1}{3} \sigma^{2} & 0 \\
0 & 0 & -\frac{1}{6} \sigma^{2}
\end{array}\right), \\
d s_{i j} & =\left(\begin{array}{lll}
0 & d \tau & 0 \\
d \tau & 0 & 0 \\
0 & 0 & 0
\end{array}\right), & d t_{i j}=\left(\begin{array}{lll}
0 & \frac{1}{3} \sigma d \tau & 0 \\
\frac{1}{3} \sigma d \tau & 0 & 0 \\
0 & 0 & 0
\end{array}\right),
\end{array}
$$

as well as $d J_{2}=d J_{3}=0$. For this special case the differential form of Eq. (14) reduces to

$$
d \epsilon_{i j}^{\prime \prime}=F\left(J_{2}, J_{3}^{2}\right)\left[P\left(J_{2}, J_{3}^{2}\right) d s_{i j}+Q\left(J_{2}, J_{3}^{2}\right) J_{3} d t_{i j}\right],
$$

where $J_{2}$ and $J_{3}$ are evaluated for an arbitrary state of pure tension. Since this state of stress satisfies the condition for a neutral change of stress $\left(d J_{2}=0\right), d \epsilon_{i j}^{\prime \prime}$ must vanish. We find then, upon substituting the values of $d s_{i j}$ and $d t_{i j}$ previously computed, that

$$
F\left(J_{2}, J_{3}^{2}\right)\left[P\left(J_{2}, J_{3}^{2}\right)+\frac{1}{3} Q\left(J_{2}, J_{3}^{2}\right) J_{3} \sigma\right]=0 .
$$

Now let us return to the finite stress-strain relation, Eq. (15), for the case of pure tension. The first component of the strain tensor (the other non-vanishing terms differ from this only by a constant factor) becomes

$$
\epsilon_{11}=\sigma+\frac{2}{3} F\left(J_{2}, J_{3}^{2}\right)\left[P\left(J_{2}, J_{3}^{2}\right) \sigma+\frac{1}{3} Q\left(J_{2}, J_{3}^{2}\right) J_{3} \sigma^{2}\right] .
$$

The invariants appearing in Eq. (20) have been evaluated for an arbitrary state of pure tension. Consequently, Eq. (20) is valid for pure tension and the second term in Eq. (21) equals zero. (A similar remark holds true for each of the other non-vanishing strain components.) Therefore, the stress-strain relation will reduce to Hooke's law for pure tension if the continuity condition is to be fulfilled. On the other hand, we have seen in Section 1 that the stress-strain law for tension need not be linear. Thus the most general finite stress-strain law coupled with Hooke's law for unloading will not be sufficiently flexible to represent a tensile test if the continuity condition is to be fulfilled. It is necessary, therefore, to turn to differential stress-strain relations if both loading and unloading are to be adequately represented.

4. Differential stress-strain relations. A system of differential stress-strain relations can be obtained from the properties discussed in Section 2 provided certain of these are rewritten in such a way as to be directly applicable in differential form. We shall assume that given the components of the stress tensor $\sigma_{i j}$ and the increments $d \sigma_{i j}$ there correspond unique strain increments $d \epsilon_{i j}$. This implies that the increment in strain, $d \epsilon_{i j}$, depends only on the state of stress at the given instant, $\sigma_{i j}$, and the increment in stress, $d \sigma_{i j}$, and is independent of the way in which this state of stress has been achieved provided only loading has taken place. In particular, we shall 
assume that this dependence is such that the increments in strain are linear functions of the increments in stress. Thus $d \epsilon_{i j}$ can be written in the form

$$
d \epsilon_{i j}=(1+\nu) d s_{i j}+(1-2 \nu) d s \delta_{i j}+c_{i j k l} d \sigma_{k l},
$$

where the fourth order tensor $c_{i j k l}$ is a function of $\sigma_{i j}$ only. For unloading, the material is assumed to satisfy the differential form of Hooke's law given in Eq. (18). Loading is supposed to take place when $d J_{2}>0$ and unloading occurs for $d J_{2}<0$. For a neutral change of stress, $d J_{2}=0$, the continuity condition requires that Eqs. (18) and (22) coincide. Consequently,

$$
c_{i j k l} d \sigma_{k l}=0 \text { whenever } d J_{2}=s_{k l} d s_{k l}=0 .
$$

Since $s_{k l}$ is a deviator, $d J_{2}$ may also be written in the form $d J_{2}=s_{k l} d \sigma_{k l}$. Thus the linear form in $d \sigma_{k l}, c_{i j k l} d \sigma_{k l}$, must vanish whenever $s_{k l} d \sigma_{k l}$ vanishes. The coefficients of $d \sigma_{k l}$ in the two forms must be proportional or

$$
c_{i j k l}=C_{i j} s_{k l},
$$

where the second order tensor $C_{i j}$ is a function of $\sigma_{k l}$ alone. The stress-strain relations then become

$$
\left.\begin{array}{lll}
d \epsilon_{i j}=(1+\nu) d s_{i j}+(1-2 \nu) d s \delta_{i j}+C_{i j} d J_{2}, & \text { when } & d J_{2} \geqq 0 ; \\
d \epsilon_{i j}=(1+\nu) d s_{i j}+(1-2 \nu) d s \delta_{i j}, & \text { when } & d J_{2} \leqq 0 .
\end{array}\right\}
$$

In a certain sense, the term $C_{i j}$ measures the permanent deformation. Indeed, let us consider the infinitesimal cycle of stress which results when first $d \sigma_{i j}$ is applied and then $-d \sigma_{i j}$. We assume, in addition, that the material is being loaded when $d \sigma_{i j}$ is applied. The permanent increment in strain $d \epsilon_{i j}^{\prime \prime}$ will then be

$$
d \epsilon_{i j}^{\prime \prime}=C_{i j} d J_{2} \text {. }
$$

Since the permanent strain is independent of a state of hydrostatic stress for pressures within the range normally encountered in testing of materials, the tensor $C_{i j}$ can only be a function of the components of the stress deviator rather than the stress tensor itself. Furthermore, there can be no permanent change in volume; that is, $d \epsilon_{i i}^{\prime \prime}=0$ or $C_{i i}=0$. Since the tensors $d \epsilon_{i j}, d s_{i j}$, and $\delta_{i j}$ are symmetric, $C_{i j}$ will also be symmetric. In addition, a reversal of the signs of all the stresses is assumed to produce a reversal of sign of all the strain increments. This implies that $C_{i j}$ must be an odd function of the stress components and thus will vanish when all the $s_{i j}$ vanish.

The material is supposed to become orthotropic under the stress $\sigma_{i j}$ in the sense that the $C_{i j}$ can be represented as a power series in the stress deviator $s_{i j}$ with scalar coefficients. These coefficients are either constants or else functions of the invariants of $s_{i j}$, i.e., functions of $J_{2}$ and $J_{3}$. It is convenient at this point to change from the subscript notation for tensors to Gibbs' notation; the tensor $C_{i j}$ will be denoted by $\mathbf{C}$ and $s_{i j}$ by $\mathbf{S}$. The multiplications indicated below are the usual matrix multiplications. Under the assumptions stated above, the tensor $\mathbf{C}$ can be written as

$$
\mathbf{C}=\sum_{n=0}^{\infty} a_{2 n+1}\left(J_{2}, J_{3}\right) \mathbf{S}^{2 n+1} \text {. }
$$

We note that only odd powers appear in Eq. (25) since $\mathbf{C}$ is assumed to be an odd 
function of the stresses. Equation (25) can be simplified further by the HamiltonCayley theorem which states that the tensor $\mathbf{S}$ must satisfy its own characteristic equation. ${ }^{3}$ For the stress deviator $\mathbf{S}$, this implies that

$$
\mathbf{S}^{3}=J_{2} \mathbf{S}+J_{3} \mathrm{I} \text {, }
$$

where I is the unit tensor. Through Eq. (26), we can reduce ${ }^{4}$ any power of $S$ greater than the second to a linear combination of $\mathbf{I}, \mathbf{S}$, and $\mathbf{S}^{2}$ with coefficients which are functions of $J_{2}$ and $J_{3}$. For example, consider the reduction of the power $\mathbf{S}^{5}$. According to Eq. (26),

$$
\mathbf{S}^{4}=J_{2} \mathbf{S}^{2}+J_{3} \mathbf{S} ;
$$

thus

$$
\mathbf{S}^{5}=J_{2} \mathbf{S}^{3}+J_{3} \mathbf{S}^{2}=J_{3} \mathbf{S}^{2}+J_{2}^{2} \mathrm{~S}+J_{2} J_{3} \mathrm{I} .
$$

In general, we can rewrite Eq. (25) as

$$
\mathbf{C}=a\left(J_{2}, J_{3}\right) \mathbf{S}^{2}+b\left(J_{2}, J_{3}\right) \mathbf{S}+c\left(J_{2}, J_{3}\right) J_{3} \mathbf{I} .
$$

We recall that $C_{i i}=0$; since $\mathbf{S}$ is a deviator, this implies that

$$
2 a\left(J_{2}, J_{3}\right) J_{2}+3 c\left(J_{2}, J_{3}\right) J_{3}=0,
$$

or

$$
c\left(J_{2}, J_{3}\right)=-\frac{2 J_{2}}{3 J_{3}} a\left(J_{2}, J_{3}\right) .
$$

Consequently,

$$
\mathbf{C}=a\left(J_{2}, J_{3}\right)\left[\mathbf{S}^{2}-\frac{2}{3} J_{2} \mathrm{I}\right]+b\left(J_{2}, J_{3}\right) \mathbf{S} .
$$

The expression appearing in square brackets is just the tensor $t_{i j}$ which was defined in Eq. (13). Returning now to the subscript notation we can write the tensor $C_{i j}$ as

$$
C_{i j}=a\left(J_{2}, J_{3}\right) t_{i j}+b\left(J_{2}, J_{3}\right) s_{i j} .
$$

A further simplification can be made by noting that $C_{i j}$ must be an odd function of the stress components. Since $J_{2}$ is even, $J_{3}$ odd, $t_{i j}$ even, and $s_{i j}$ odd, we must have

$$
C_{i j}=p\left(J_{2}, J_{3}^{2}\right) s_{i j}+q\left(J_{2}, J_{3}^{2}\right) J_{3} t_{i j}
$$

Thus the complete differential stress-strain relations can be written in the form ${ }^{b}$

$$
\left.\begin{array}{lr}
d \epsilon_{i j}=(1+\nu) d s_{i j}+(1-2 \nu) d s \delta_{i j}+\left[p\left(J_{2}, J_{3}^{2}\right) s_{i j}+q\left(J_{2}, J_{3}^{2}\right) J_{3} t_{i j}\right] d J_{2} \text { when } d J_{2} \geqq 0 \\
d \epsilon_{i j}=(1+\nu) d s_{i j}+(1-2 \nu) d s \delta_{i j}, & \text { when } d J_{2} \leqq 0 .
\end{array}\right\}
$$

5. Further study of the stress strain relations. Experimental determination. In this section we shall discuss the relation between the differential form of the stressstrain relation (cf. (27))

${ }^{8}$ M. Bôcher, Introduction to higher algebra, The Macmillan Co., New York, 1907, p. 296.

4 This technique has been used recently by Marcus Reiner, Am. J. Math. 67, 350-362 (1945) and W. Prager, loc. cit.

S These relations contain, as special cases, the stress-strain laws developed by W. Prager, Proc. Fifth International Congress of Applied Mechanics, Cambridge, Mass., 1938, pp. 234-237, and by J. H. Laning in an unpublished paper (1942). 


$$
d \epsilon_{i j}^{\prime \prime}=\left\{p\left(J_{2}, J_{3}^{2}\right) s_{i j}+q\left(J_{2}, J_{3}^{2}\right) J_{3} t_{i j}\right\} d J_{2}, \quad d J_{2} \geqq 0
$$

and the integral form

$$
\epsilon_{i j}^{\prime \prime}=F\left(J_{2}, J_{3}^{2}\right)\left[P\left(J_{2}, J_{3}^{2}\right) s_{i j}+Q\left(J_{2}, J_{3}^{2}\right) J_{3} t_{i j}\right],
$$

which holds only when the ratios of the principal stresses are kept constant during the loading process, i.e., if

$$
s_{i j}=k s_{i j}^{(0)},
$$

where $s_{i j}^{(0)}$ is fixed while $k$ is the scalar variable. We shall then show how a series of tests necessary to establish the Lode diagram will be sufficient to determine the stressstrain relations completely. First of all, it is convenient to bring out the homogeneity properties in the relations (28) and (14) by introducing the symbols

$$
\alpha=J_{3}^{2} / J_{2}^{3}, \quad \gamma_{i j}=J_{3} t_{i j} / J_{2}^{2},
$$

where $\alpha$ is dimensionless, while $\gamma_{i j}$ has the same dimensions as $J_{2}$. The relation (14) can be written in the form

where

$$
\epsilon_{i j}^{\prime \prime}=\lambda\left(J_{2}, \alpha\right)\left\{s_{i j}+\beta(\alpha) \gamma_{i j}\right\}
$$

$$
\lambda\left(J_{2}, \alpha\right) \equiv F\left(J_{2}, J_{3}^{2}\right) P\left(J_{2}, J_{3}^{2}\right), \quad \beta(\alpha) \equiv J_{2}^{2} Q\left(J_{2}, J_{3}^{2}\right) / P\left(J_{2}, J_{3}^{2}\right) .
$$

Note that $\beta$ is independent of $J_{2}$, because of the homogeneity relation between $P$ and $Q$ established in Section 2.

With a similar change of notation, the relation (28) can be written as

$$
d \epsilon_{i j}^{\prime \prime}=G\left(J_{2}, \alpha\right)\left\{s_{i j}+\beta^{\prime}(\alpha) \gamma_{i j}\right\} d J_{2}, \quad d J_{2} \geqq 0
$$

where

$$
G\left(J_{2}, \alpha\right) \equiv p\left(J_{2}, J_{3}^{2}\right), \quad \beta^{\prime}(\alpha) \equiv J_{2}^{2} q\left(J_{2}, J_{3}^{2}\right) / p\left(J_{2}, J_{3}^{2}\right) .
$$

Since we did not establish a homogeneity relation between $p$ and $q$, we cannot immedilately conclude that $\beta^{\prime}$ is independent of $J_{2}$. However, we shall see immediately that this is true and that indeed

$$
\beta^{\prime} \equiv \beta .
$$

We shall also show that $G\left(J_{2}, \alpha\right)$ may be obtained from $\lambda\left(J_{2}, \alpha\right)$ by the relation

$$
G\left(J_{2}, \alpha\right)=\frac{\lambda}{2 J_{2}}+\frac{\partial \lambda}{\partial J_{2}} .
$$

The relations (35) and (36) will then determine the differential relation (33) completely once the integral relation (31) is known by a series of experiments of the special type (29). It is to be noted that the functions $G\left(J_{2}, \alpha\right)$ and $\beta(\alpha)$ in (33) determined through the use of (31) will by no means restrict the application of (33) to processes connected in any manner with (29).

To establish the relations (35) and (36), consider the application of (31) and (33) to a process of the type (29). Let $d \epsilon_{i j}^{\prime \prime}$ be the change in $\epsilon_{i j}^{\prime \prime}$ corresponding to a change $d k$. Then 


$$
d J_{2}=2 J_{2} \frac{d k}{k}, \quad d \alpha=0 ;
$$

and (31) gives

while (33) yields

$$
d \epsilon_{i j}^{\prime \prime}=\frac{\partial \lambda}{\partial J_{2}} d J_{2}\left\{s_{i j}+\beta \gamma_{i j}\right\}+\lambda\left\{s_{i j}+\beta \gamma_{i j}\right\} \frac{d k}{k},
$$

$$
d \epsilon_{i j}^{\prime \prime}=G\left(J_{2}, \alpha\right)\left\{s_{i j}+\beta^{\prime} \gamma_{i j}\right\} d J_{2} .
$$

Equating coefficients of $s_{i j}$ and $\gamma_{i j}$, we obtain the relations (35) and (36).

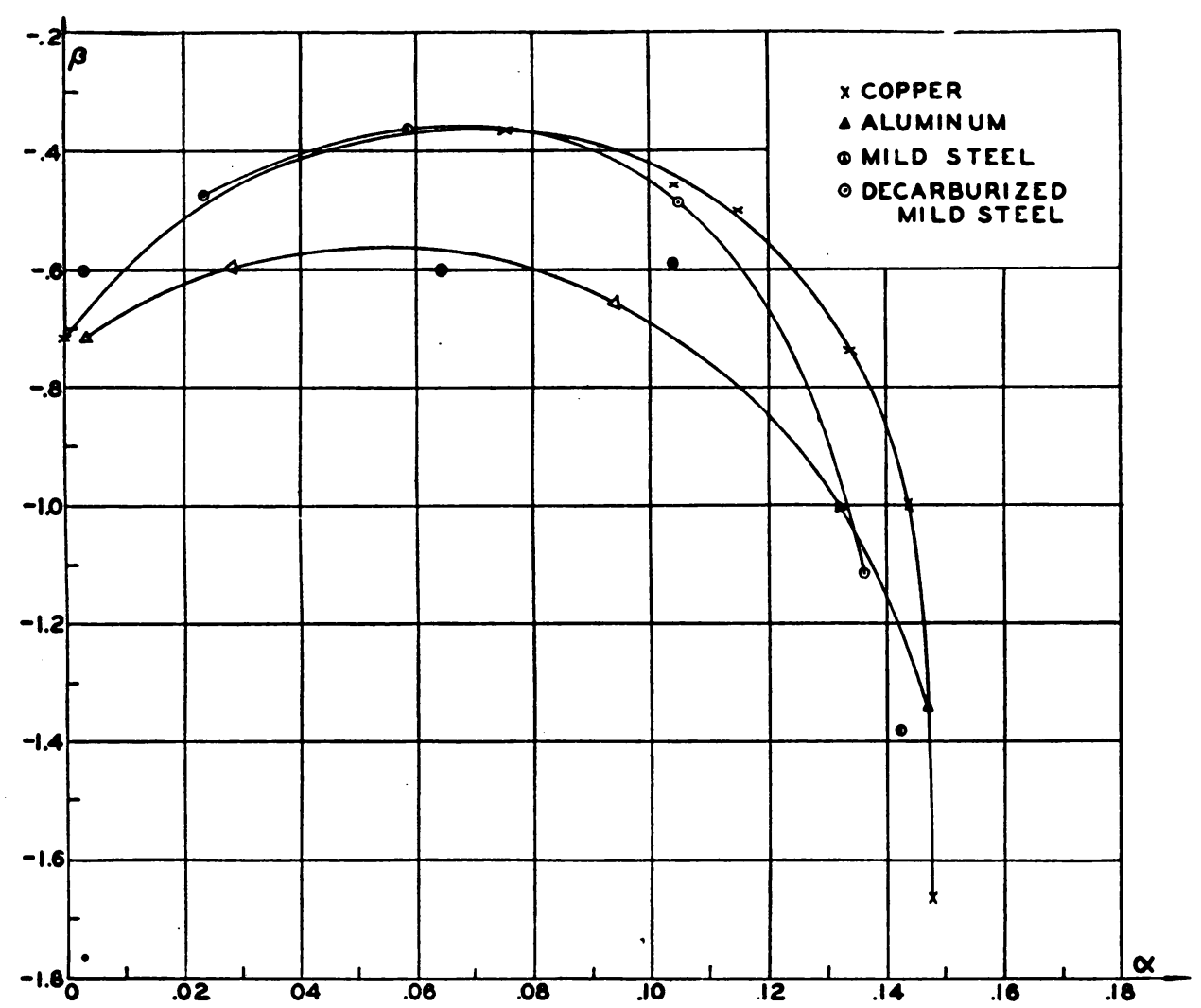

Fig. 2a. The $\alpha-\beta$ diagram (Eq. (38)) of the experimental results of Taylor and Quinney for copper, aluminum, mild steel and decarburized mild steel. The data for mild steel are too scattered for a definite curve to be drawn.

The experimental determination of the stress strain relations can then be reduced to that of (31) alone. This can be done by a series of tests of the type (29), which is of the class described by Lode, ${ }^{6}$ Taylor-Quinney ${ }^{7}$ and Hohenemser-Prager. ${ }^{8}$ Indeed,

- W. Lodge, Forschungsarbeiten a.d. Gebiete d. Ingenieurwesens, No. 303, VDI-Verlag, Berlin, 1928.

${ }^{7}$ G. I. Taylor and H. Quinney, Phil. Trans. Roy. Soc. London (A) 230, 323-362 (1931).

8 K. Hohenemser and W. Prager, Z. angew. Math. Mech. 12, 1-14 (1932). An English translation of this paper is available as R.T.P. Translation No. 2468 (Durand Reprinting Committee, in care of California Institute of Technology, Pasadena 4, Calif.). 
the relation $\beta(\alpha)$ is merely another presentation of Lode's diagram. It can be easily verified that $\alpha, \beta$ are related to Lode's parameters ${ }^{\vartheta} \mu$ and $\nu$ by the relations

$$
\left.\begin{array}{rl}
\alpha & =\frac{4}{27} \frac{\mu^{2}\left(9-\mu^{2}\right)^{2}}{\left(3+\mu^{2}\right)^{3}}, \\
\beta & =\frac{9\left(3+\mu^{2}\right)^{2}}{2\left(9-\mu^{2}\right)} \frac{1-\nu / \mu}{\mu^{2}(1+2 \nu / \mu)-3} \cdot
\end{array}\right\}
$$

This new system has the advantage that $\beta$ gives directly the extent of deviation from "von Mises' second hypothesis" discussed by Taylor and Quinney, which is equivalent to putting $\beta \equiv 0$. Indeed, one principal aim of Taylor and Quinney is to find out

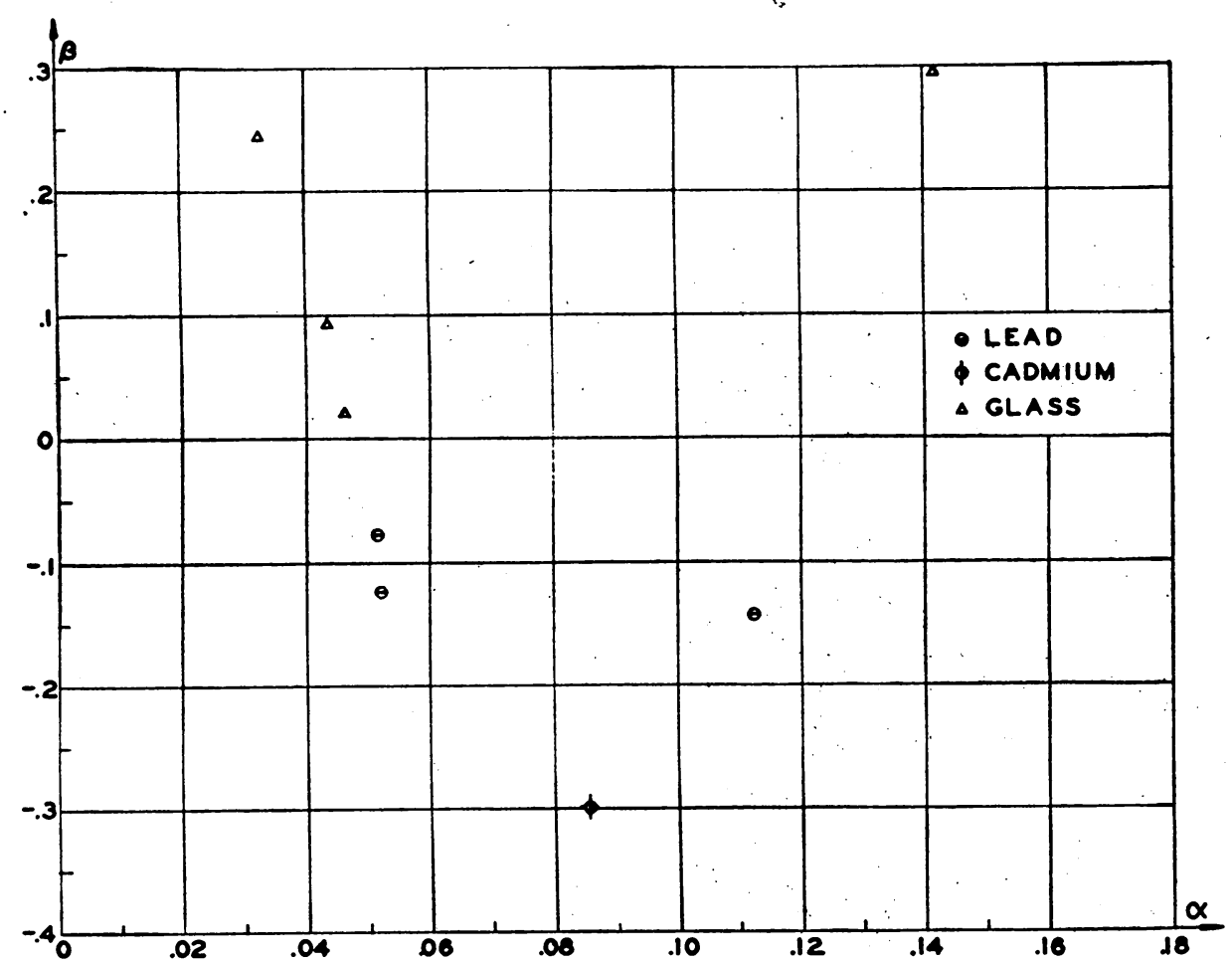

FIG. 2b. The $\alpha-\beta$ diagram (Eq. (38)) of the experimental results of Taylor and Quinney for lead, cadmium and glass. The data are too few to allow any curve to be drawn.

this extent and is therefore to determine the value of $\beta$. Figs. 2(a) and 2(b) show the results of Taylor and Quinney converted into the $(\alpha, \beta)$ diagram. This diagram reveals any experimental error more strongly, since $\beta$ is essentially related to the slope of the $(\mu, \nu)$ curve; e.g.,

9 W. Lode, loc. cit., pp. 1 and 12. 


$$
\begin{aligned}
& \frac{d \nu}{d \mu}=\frac{9-4 \beta}{9+2 \beta} \text { for } \mu \rightarrow 1, \\
& \frac{d \nu}{d \mu}=1+\frac{2 \beta}{3} \text { for } \mu=0 .
\end{aligned}
$$

It should be noted in passing that $\alpha$ must satisfy the inequality

$$
0<\alpha<4 / 27
$$

to insure real values of $\mu$.

Having determined $\beta(\alpha)$ from (38), we may determine $\lambda\left(J_{2}, \alpha\right)$ by noting that (cf. (31))

$$
I_{2} \equiv \frac{1}{2} \epsilon_{i j}^{\prime \prime \prime} \epsilon_{i j}^{\prime \prime}=\lambda^{2} J_{2}\left\{1+3 \alpha \beta+\frac{1}{3} \alpha^{2} \beta^{2}\right\} \text {. }
$$

For each loading process given by (29) the value of $\alpha$ is fixed, and (40) gives the dependence of $\lambda^{2}$ on $J_{2}$ if $I_{2}$ is determined for given values of $J_{2}$. A series of tests with different principal axes will then give the further dependence of $\lambda$ on $\alpha$.

6. Concluding remarks. In closing, we note some of the limitations of the stressstrain relations developed in this paper. It has been pointed out previously that these equations have been developed to cover the case of one loading followed by at most one unloading. This restriction is quite essential, for relations (27) are not applicable for a second loading. For example, if we consider a simple tensile test, the stress-strain diagram obtained from (27) for the second loading would be a mere translation of the diagram for the first loading. This does not agree with the experimental results. Secondly, we note that these equations apply only to metals which exhibit strain-hardening. They are not applicable, for example, to materials which yield under constant shearing stress or satisfy von Mises' yield condition, $J_{2}=$ const.

It is hoped that the results presented here will provoke experimental work to test their validity. Among the various features which should be tested are two assumptions made in developing the differential stress-strain laws. The first hypothesis (cf. Section 4) states that the increments in strain are uniquely determined by the components of the stress tensor $\sigma_{i j}$ and the increments $d \sigma_{i j}$ without reference to the previous history of loading provided only one loading has taken place followed by at most one unloading. The range in which such a hypothesis is valid should be explored empirically. Secondly, the assumption involved in the transition from Eqs. (24) to (25) should be examined carefully. According to these two relations, the principal axes of the increment in permanent strain $d \epsilon_{l j}^{\prime \prime}$ will coincide with the principal axes of the existing state of stress $s_{i j}$ independent of the increments in stress $d \sigma_{i j}$, provided only loading takes place. This conclusion should be tested by experiment. 